\title{
Severe Cardiac Valve Stenosis
}

National Cancer Institute

\section{Source}

National Cancer Institute. Severe Cardiac Valve Stenosis. NCI Thesaurus. Code C100013.

The cardiac valve orifice is abnormally narrow, to a severe degree. (ACC) 